\title{
Fisiopatología y manifestaciones bucales de la enfermedad de Parkinson: Una revisión actualizada
}

\section{Pathophysiology and oral manifestations of Parkinson's disease: A review update}

\author{
Harris Ricardo J*, Fortich Mesa N**, Díaz Caballero A**
}

\section{RESUMEN}

La enfermedad de Parkinson es un trastorno neurovegetativo, crónico y lentamente progresivo del sistema nervioso central que se caracteriza por temblor en reposo, rigidez muscular, lentitud de movimientos e inestabilidad postural. Para brindar una atención competente a los pacientes los odontólogos deben entender la fisiopatología, complicaciones y manifestaciones bucales de la enfermedad, al instaurar el plan de tratamiento, siendo importante un enfoque multidisciplinario para el manejo.

En el presente artículo se describen los aspectos fisiopatológicos de la enfermedad de Parkinson, manifestaciones bucales y recomendaciones para el manejo bucal.

Palabras clave: Enfermedad de Parkinson, higiene bucal, xerostomía, anestésicos locales. (Decs Bireme).

\section{SUMMARY}

Parkinson's disease is one of a group of relatively common, progressive, neurological disorder extrapyramidal diseases characterized by rigidity and tremor. Dental management of individuals with Parkinson's is a multifaceted challenge involving areas of preventive, restorative, and prosthetic dentistry. To provide competent care to patients dentists must understand the pathophysiology, complications, and oral manifestations of the disease, to establish the treatment plan. The successful management of the disease requires a multi-disciplinary approach. This article describes the pathophysiology of Parkinson's disease, oral manifestations and dental management recommendations.

Key words: Parkinson Disease, oral hygiene, xerostomía, anesthetics local. (Mesh Database).

Fecha de recepción: 20 de junio de 2012.

Aceptado para publicación: 5 de julio de 2012.

* Odontólogo. Universidad del Sinú. Especialista en Estomatología y Cirugía Oral. Universidad de Cartagena. Docente Corporación Universitaria Rafael Núñez.

** Odontóloga. Universidad Javeriana. Especialista en Endodoncia. Universidad de Cartagena. Docente Corporación Universitaria Rafael Núñez.

*** Odontólogo. Universidad de Cartagena. Especialista en Periodoncia Universidad Javeriana. Profesor titular de la Universidad de Cartagena. Director Grupo de investigaciones GITOUC.

Harris Ricardo J, Fortich Mesa N, Díaz Caballero A. Fisiopatología y manifestaciones bucales de la enfermedad de Parkinson: Una revisión actualizada. Av. Odontoestomatol 2013; 29 (3): 151-157. 


\section{INTRODUCCIÓN}

La enfermedad de Parkinson (EP) es una patología neurodegenerativa del sistema nervioso central, descrita por primera vez en 1817 por James Parkinson, quien la denominó parálisis temblorosa y posteriormente llevaría su nombre $(1,2)$, se caracteriza por el deterioro progresivo de las neuronas en la sustancia negra del cerebro, generando una disminución de la dopamina (3).

La dopamina cumple funciones neurotransmisoras responsable de transmitir señales, su disminución en el los núcleos basales, produce una disfunción en la regulación de las principales estructuras cerebrales implicadas en el control del movimiento, las alteraciones motoras están relacionadas con la perdida de secreción de la dopamina por los centros dopaminérgicos y un aumento de la actividad en las vías de la acetilcolina $(4,5)$.

Los trastornos del movimiento inician gradualmente, la edad media del comienzo de la enfermedad se presenta a los 55 años $(6,7)$. Los signos y síntomas principales son temblor, rigidez, bradicinesia y pérdida del equilibrio o inestabilidad postural; el temblor comienza de manera gradual afectando las manos, brazos, piernas y la mandíbula, aumenta con la fatiga, tensión emocional y desaparece durante el sueño, los músculos permanecen tensos y contraídos originando dolor, se presenta dificultad en la marcha, disminución del parpadeo y facies en máscara, durante la deambulación dan pasos cortos y no se presenta el braceo de las extremidades superiores (8-11).

La causa de esta enfermedad es desconocida, pero se reconoce cada vez más la importancia de la genética, varios genes han sido ligados a la enfermedad, el primero identificado fue la alfa-sinucleína, una proteína que regula las comunicaciones entre neuronas en las sinapsis y forma los cuerpos de Lewy $(12,13)$.

Una mutación en el gen alfa-sinucleína causa una forma temprana y hereditaria de la enfermedad; mientras que los cuerpos de Lewy se presentan en los casos esporádicos (14-16). Otros genes ligados a la EP relacionados con el inicio temprano son la parkina, normalmente ayuda a las células a descom- poner y reciclar las proteínas, el DJ-1 ayuda a proteger las células del estrés oxidativo, el PINK1 codifica a una proteína activa en las mitocondrias, aumenta la susceptibilidad al estrés celular (17-21).

\section{DIAGNÓSTICO Y PRONÓSTICO}

El diagnóstico de la EP se basa en la historia clínica y en un examen neurológico, ya que no existen pruebas de laboratorio, marcadores bioquímicos o anatomopatológico, que ayuden al diagnóstico preciso de la enfermedad (22-24). Los signos y síntomas tempranos son difíciles de percibir, comúnmente pasan desapercibidos o pueden ser descartados como los efectos del envejecimiento normal (25). Estudios imagenológicos como tomografía computarizada y resonancia magnética se realizan con el fin de descartar otras enfermedades, ya que en la EP generalmente aparecen normales (26).

La EP es crónica y empeora con el tiempo, la evolución de los síntomas en algunas personas puede ser más rápido, siendo difícil predecir qué curso seguirá la enfermedad (27), es importante utilizar escalas estandarizadas de valoración que permitan monitorizar el seguimiento y el estado clínico como la clasificación de estadios de Hoehn y Yahr (28) (Tabla 1).

\section{TRATAMIENTO}

La terapéutica farmacológica abarca tres categorías, en la primera los fármacos actúan directa o indirectamente aumentando el nivel de dopamina en el cerebro como la levodopa, apomorfina, bromocriptina, pramipexol entre otros $(29,30)$, la segunda categoría afecta a otros neurotransmisores con el fin de aliviar algunos síntomas de la enfermedad como temblores y la rigidez muscular, siendo útiles los anticolinérgicos como el trihexifenidil, benzotropina y etopropazina, finalmente en la tercera categoría los fármacos que controlan los síntomas no motores como antidepresivos, antihipertensivos, antisicóticos atípicos etc. (31-33). La terapéutica quirúrgica es aplicada en las personas con enfermedad avanzada para quienes el tratamiento farmacológico no es suficiente (34). 


\section{TABLA 1.- ESTADIOS DE HOEHN Y YAHR PARA LA PROGRESIÓN DE LA ENFERMEDAD DE PARKINSON}

\section{Estadio 1:}

- Signos y síntomas leves en un solo lado.

- Síntomas molestos pero no incapacitantes.

- Presencia de síntomas con temblor en alguna extremidad.

\section{Estadio 2:}

- Síntomas bilaterales.

- Mínima discapacidad.

- La marcha y la postura están afectadas.

\section{Estadio 3:}

- Significante enlentecimiento de los movimientos corporales.

- Dificultad para mantener el equilibrio tanto de pie como al andar.

- Disfunción generalizada moderadamente severa.

\section{Estadio 4:}

- Síntomas severos.

- Todavía puede andar cierto recorrido.

- Rigidez y bradicinesia.

\section{Estadio 5:}

- Estadio caquéctico.

- Invalidez total.

- No puede andar ni mantenerse de pie, si no tiene ayuda.

\section{MANIFESTACIONES BUICALES}

La condición progresiva de la enfermedad conduce a restricciones en la movilidad y rigidez muscular, lo cual lleva a problemas en el mantenimiento de la una adecuada salud oral (35). En la mayoría de los afectados la disartria es característica y pueden utilizar un tono de voz muy suave o repetir las palabras $(36,37)$ el temblor en la musculatura facial y los movimiento en la mandibular son notorios, así como la rigidez en alguno de estos músculos, también presentan dificultad en la deglución ya que la musculatura involucrada podrían funcionar con menor efi- ciencia, permitir retención de alimentos en la orofaringe y aumentar el riesgo de aspiración por la vía aérea $(38,39)$; el babeo es frecuente Kalf et al, realizaron un estudio en donde revisaron sistemáticamente la literatura de acuerdo con la prevalencia del babeo, encontraron ocho estudios con tasas de prevalencia que van de 32 a $74 \%$ y la mayor presentación fue nocturna, afirmando que el babeo puede estar presente en la mitad de los pacientes que padecen EP (40).

Otra característica es la disminución del flujo salival relacionada ampliamente con la terapia farmacológica, aumentando el riesgo de presentar caries y la aparición de infecciones como la candidiasis, el sabor amargo o alteración en la percepción del gusto, siendo un efecto secundario de la medicación especialmente la levodopa $(41,42)$.

La lengua puede irritarse y aparecer ulceraciones (43), Clifford et al, determinaron la prevalencia del síndrome de boca ardiente en pacientes con EP, en donde $24 \%$ de los participantes en el estudio lo manifestaron y describen que la razón es incierta, pero la relacionan con la terapia farmacología y el aumento en el tono muscular (44).

El edentulismo es significativamente mayor debido a la falta de control en los movimientos musculares, el cual dificulta una buena higiene oral y aumenta el riesgo de enfermedad periodontal avanzada (45), Schwaz et al, determinaron el estado periodontal en pacientes con EP y concluyen que los problemas de higiene bucal contribuyen al aumento de la patologías periodontales (46). También pueden producirse patologías en la articulación temporomandibular, atrición severa y en pacientes con prótesis dental se presenta dificultad para retenerlas en la posición correcta (47).

\section{RECOMENDACIONES PARA EL MANEJO BUCAL}

La atención odontológica integral es parte del tratamiento interdisciplinario, los odontólogos deben estar atentos del desarrollo de las manifestaciones en la cavidad oral para implementar un buen tratamiento o control de las mismas, es primordial an- 
tes de instaurar alguna terapéutica consultar con el médico tratante para conocer en qué etapa de la patología se encuentra y si presenta otras enfermedades que puedan modificar el tratamiento odontológico (48).

Los temblores y la rigidez muscular pueden generar dificultad en el examen clínico intrabucal, es importante que las citas sean cortas y se programen en la mañana, noventa minutos después de la administración del fármaco antiparkinsoniano, ya que existen reportes que afirman buena colaboración por parte del paciente en este periodo $(41,49)$; la utilización de los bloques de mordida es esencial para mantener la boca abierta y un succionador de alta potencia para proteger las vías aéreas (50).

La dificultad en la retención y posición correcta de la prótesis dental es común, Packer et al, investigaron el impacto de los implantes dentales en la calidad de vida en personas con EP, de acuerdo con los resultados destacaron mejora significativa en los ámbitos de la alimentación y satisfacción con la prótesis implantosoportada, afirmando que esta superaba algunas de las dificultades, ya que son más estables en comparación con las convencionales (51).

La sialorrea es otra de las manifestaciones en la cual se han utilizado muchos medicamentos para su tratamiento sin mayor efectividad, en un estudio Friedman et al, aplicaron inyecciones locales de toxina botulínica tipo A en las glándulas salivales parótida de 11 pacientes con sialorrea, después de aplicada la terapéutica se observó en los controles disminución de la excreción salival y afirman que la toxina botulínica tipo A es una alternativa de tratamiento eficaz y segura, ya que no se notaron efectos secundarios (52). Ondo et al, determinaron la efectividad de la toxina botulínica tipo B en 16 pacientes con sialorrea, aplicaron 1.000 unidades en cada glándula parótida y 250 unidades en cada glándula submandibular, en cuanto a los resultados de la terapéutica destacan su eficacia para la sialorrea (53).

Los pacientes con EP que reciban levodopa y entacapona se les debe limitar la administración de lidocaína 1:100.000 con epinefrina al dos por ciento a un número de tres carpules para evitar la taquicardia y la hipertensión $(54,55)$; los enfermos que estén bajo tratamiento con rasagilina (inhibidor de monoamina oxidasa) no se les debe administrar anestésicos locales que contengan fármacos simpaticomiméticos vasoconstrictores como la epinefrina o levonordefrina, ya que pueden provocar una crisis hipertensiva, hiperpirexia, diaforesis y hemorragia subaracnoide; la rasagilina debe suspenderse dos semanas antes de cualquier cirugía que requiera anestesia general y en caso de procedimientos de emergencia, pueden emplearse benzodiazepinas, mivacurium, fentanilo, morfina o codeína, con cautela (56).

Existe mayor riesgo de presentar caries en las personas que padecen EP debido a la disminución del control de la capacidad motriz, por la ingesta de alimentos blandos que tiene una buena adherencia a las estructuras dentales, la baja ingesta de líquidos y en algunos casos disminución del fluido salival (57); Müller et al, realizaron un estudio donde investigaron la salud oral de 101 pacientes con EP y los compararon con un grupo control, en los resultados aquellos que presentaron peor estado de salud oral, frecuencia baja de cepillado diario y el tiempo más largo desde la última visita al odontólogo fueron los pacientes con EP y reportaron que la instrucción por parte del profesional en el área de la salud bucal, en cuanto a una buena higiene oral es fundamental para la prevención de patologías orales y es recomendable la utilización del cepillo eléctrico (58).

\section{CONCLUSIONES}

La EP no tiene una causa única, existiendo casos familiares con anomalías genéticas conocidas y otros casos en los que una conjunción de factores genéticos y ambientales serían los responsables de la muerte neuronal.

La mayoría de las complicaciones bucales surgen por la incapacidad del paciente para llevar a cabo la higiene bucal de rutina y los efectos secundarios de los fármacos empleados en la terapéutica; siendo importante aumentar la frecuencia de la consulta odontológica para prevenir patologías bucales y crear conciencia al paciente en mantener una buena higiene oral. 


\section{BIBLIOGRAFÍA}

1. Dirks SJ, Paunovich ED, Terezhalmy GT, Chiodo LK. The patient with Parkinson's disease. Quintessence Int 2003 May;34(5):379-93.

2. Raudino F. The Parkinson disease before James Parkinson. Neurol Sci 2011 Oct 18. [Epub ahead of print].

3. Boczarska-Jedynak M, Opala G. Sleep disturbances in Parkinson's disease. Neurol Neurochir Pol 2005;Sep-Oct;39(5):380-8.

4. Martin HL, Teismann P. Glutathione-a review on its role and significance in Parkinson's disease. FASEB J 2009;Oct;23(10):3263-72.

5. Derkinderen P, Rouaud T, Lebouvier T, Bruley des Varannes S, Neunlist M, De Giorgio R. Parkinson disease: the enteric nervous system spills its guts. Neurology 2011;Nov 8;77(19):1761-7.

6. Dorsey ER, Constantinescu R, Thompson JP, et al. Projected number of people with Parkinson disease in the most populous nations, 2005 through 2030. Neurology 2007;Jan 30;68(5): 384-6.

7. Baumann CR. Epidemiology, diagnosis and differential diagnosis in Parkinson's disease tremor. Parkinsonism Relat Disord 2012;Jan; 18 Suppl 1:S90-2.

8. Hobson DE. Clinical manifestations of Parkinson's disease and parkinsonism. Can J Neurol Sci 2003 Mar;30 Suppl 1:S2-9.

9. Tison F. Diagnostic criteria of parkinsonian syndromes. Rev Neurol (Paris) 2003;May;159(5 Pt 2):3S19-23.

10. Mehndiratta M, Garg RK, Pandey S. Nonmotor symptom complex of Parkinson's disease-an under-recognized entity. J Assoc Physicians India 2011;May;59:302-8.

11. Diamond A, Jankovic J. Treatment of advanced Parkinson's disease. Expert Rev Neurother 2006; Aug;6(8):1181-97.
12. Hofer A, Gasser T. New aspects of genetic contributions to Parkinson's disease. J Mol Neurosci 2004;24(3):417-24.

13. Cookson MR. The biochemistry of Parkinson's disease. Annu Rev Biochem 2005;74:29-52.

14. van de Berg WD, Hepp DH, Dijkstra AA, Rozemuller JA, Berendse HW, Foncke E. Patterns of alphasynuclein pathology in incidental cases and clinical subtypes of Parkinson's disease. Parkinsonism Relat Disord 2012;Jan;18 Suppl 1:S28-30.

15. Jellinger KA. A critical evaluation of current staging of alpha-synuclein pathology in Lewy body disorders. Biochim Biophys Acta 2009; Jul; 1792(7):730-40.

16. Gasser T. Update on the genetics of Parkinson's disease. Mov Disord 2007;Sep;22 Suppl 17: S343-50.

17. Bardien S, Lesage S, Brice A, Carr J. Genetic characteristics of leucine-rich repeat kinase 2 (LRRK2) associated Parkinson's disease. Parkinsonism Relat Disord 2011;Aug;17(7):5018.

18. Bonifati V. Genetics of Parkinson's disease. Minerva Med 2005 Jun;96(3):175-86.

19. Mizuno Y. Progress in the basic and clinical aspects of Parkinson's disease. Rinsho Shinkeigaku 2004 Nov;44(11):741-50.

20. Choi JY, Park CS, Kim DJ, Cho MH, Jin BK, Pie JE, Chung WG. Prevention of nitric oxide-mediated 1-methyl-4-phenyl-1,2,3,6-tetrahydropyridineinduced Parkinson's disease in mice by tea phenolic epigallocatechin 3-gallate. Neurotoxicology 2002; Sep;23(3):367-74.

21. Hattori N. Etiology and pathogenesis of Parkinson's disease: from mitochondrial dysfunctions to familial Parkinson's disease. Rinsho Shinkeigaku 2004; Apr-May;44(4-5):241-62.

22. Pahwa R, Lyons KE. Early diagnosis of Parkinson's disease: recommendations from diagnostic 
clinical guidelines. Am J Manag Care 2010 Mar; 16 Suppl Implications:S94-9.

23. Brooks DJ. Parkinson's disease: Diagnosis. Parkinsonism Relat Disord 2012; Jan; 18 Suppl 1: S31-3.

24. Alrefai A, Habahbih M, Alkhawajah M, Darwish M, Batayha W, Khader Y, El-Salem K. Prevalence of Parkinson's disease in Northern Jordan. Clin Neurol Neurosurg 2009;Dec;111 (10):812-5.

25. Poewe W, Wenning G. The differential diagnosis of Parkinson's disease. Eur J Neurol 2002 Nov;9 Suppl 3:23-30.

26. Savoiardo M. Differential diagnosis of Parkinson's disease and atypical parkinsonian disorders by magnetic resonance imaging. Neurol Sci 2003 May;24 Suppl 1:S35-7.

27. Fernández HH. Updates in the medical management of Parkinson disease. Cleve Clin J Med 2012;Jan;79(1):28-35.

28. Hoehn MM, Yahr MD. Parkinsonism: onset, progression, and mortality 1967 . Neurology 2001;Nov;57(10 Suppl 3):S11-26.

29. Noble C. Modes of drug delivery used to manage Parkinson's disease. Nurs Times 2006; Aug 8-14; 102(32):30-2.

30. Rezak M. Current pharmacotherapeutic treatment options in Parkinson's disease. Dis Mon 2007 Apr; 53(4):214-22.

31. Münchau A, Bhatia KP. Pharmacological treatment of Parkinson's disease. Postgrad Med J 2000;Oct;76(900):602-10.

32. Cranwell-Bruce LA. Drugs for Parkinson's disease. Medsurg Nurs 2010;Nov-Dec;19(6): 347-9.

33. Chen JJ, Swope DM. Pharmacotherapy for Parkinson's disease. Pharmacotherapy 2007;Dec; 27(12 Pt 2):161S-173S.
34. Guridi J, Rodríguez M, Manrique m. Tratamiento quirúrgico de la enfermedad de Parkinson. Neurocirugía 2004;15:5-16.

35. Nakayama Y, Washio M, Mori M. Oral health conditions in patients with Parkinson's disease. J Epidemiol 2004;Sep;14(5):143-50.

36. Alexander RE, Gage TW. Parkinson's disease: an update for dentists. Gen Dent 2000;Sep-Oct;48 (5):572-80.

37. Fiske J, Hyland K. Parkinson's disease and oral care. Dent Update 2000;Mar;27(2):58-65.

38. Wierink CD, Bots-van 't Spijker PC, de Baat C. Specialties in dentistry. Salivary flow and swallowing in Parkinson's disease. Ned Tijdschr Tandheelkd 2006;Dec;113(12):502-5.

39. Bakke M, Larsen SL, Lautrup C, Karlsborg M. Orofacial function and oral health in patients with Parkinson's disease. Eur J Oral Sci 2011;Feb;119 (1):27-32.

40. Kalf JG, de Swart BJ, Borm GF, Bloem BR, Munneke M. Prevalence and definition of drooling in Parkinson's disease: a systematic review. J Neurol 2009;Sep;256(9):1391-6.

41. Friedlander $\mathrm{AH}$, Mahler $M$, Norman KM, Ettinger RL. Parkinson disease: systemic and orofacial manifestations, medical and dental management. J Am Dent Assoc 2009;Jun;140(6):658-69.

42. Proulx M, De Courval F, Wiseman M, Panisset M. Salivary production in Parkinson's disease. Mov Disorders. 2005 Feb; 20 (2) :204-7.

43. Van Lieshout PH, Steele CM, Lang AE. Tongue control for swallowing in Parkinson's disease: effects of age, rate, and stimulus consistency. Mov Disord. 2011 Aug 1;26(9):1725-9.

44. Clifford TJ, Warsi MJ, Burnett CA, Lamey PJ. Burning mouth in Parkinson's disease sufferers. Gerodontology. 1998;15(2):73-8.

45. Einarsdóttir ER, Gunnsteinsdóttir H, Hallsdóttir MH, Sveinsson S, Jónsdóttir SR, Olafsson VG, 
Bragason TH, Saemundsson SR, Holbrook WP. Dental health of patients with Parkinson's disease in Iceland. Spec Care Dentist 2009;May-June;29 (3):123-7.

46. Schwarz J, Heimhilger E, Storch A. Increased periodontal pathology in Parkinson's disease. J Neurol 2006;May;253(5):608-11.

47. Makzoume JE. Complete denture prosthodontics for a patient with Parkinson's disease using the neutral zone concept: a clinical report. Gen Dent 2008;May-Jun;56(4):12-6.

48. Hanaoka A, Kashihara K. Increased frequencies of caries, periodontal disease and tooth loss in patients with Parkinson's disease. J Clin Neurosci 2009;Oct;16(10):1279-82.

49. Jolly DE, Paulson RB, Paulson GW, Pike JA. Parkinson's disease: a review and recommendations for dental management. Spec Care Dentist 1989 May-Jun;9(3):74-8.

50. Silvestre F, Plaza A. Odontología en pacientes especiales. España. Universidad de valencia; 2007:293-6.

51. Packer M, Nikitin V, Coward T, Davis DM, Fiske J. The potential benefits of dental implants on the oral health quality of life of people with Parkinson's disease. Gerodontology 2009;Mar;26(1):11-8.

52. Friedman A, Potulska A. Botulinum toxin for treatment of parkinsonian sialorrhea. Neurol Neurochir Pol 2001;35 Suppl 3:23-7.
53. Ondo WG, Hunter C, Moore W. A double-blind placebo-controlled trial of botulinum toxin $\mathrm{B}$ for sialorrhea in Parkinson's disease. Neurology 2004;Jan 13;62(1):37-40.

54. Ishihara A, Miyachi T, Nakamura T, Ohtsuki T, Kimura Y, Kihira K, Yamawaki T, Matsumoto M. Relationship between 3-O-methyldopa and the clinical effects of entacapone in advanced Parkinson's disease. Hiroshima J Med Sci 2011; Sep;60(3):57-62.

55. Kalenka A, Hinkelbein J. Anaesthesia in patients with Parkinson's disease. Anaesthesist 2005; Apr;54(4):401-9.

56. Guay DR. Rasagiline (TVP-1012): a new selective monoamine oxidase inhibitor for Parkinson's disease. Am J Geriatr Pharmacother 2006;Dec; 4(4):330-46.

57. Fukayo S, Nonaka K, Shimizu T, Yano E. Oral health of patients with Parkinson's disease: factors related to their better dental status. Tohoku J Exp Med 2003;Nov;201(3):171-9.

58. Müller T, Palluch R, Jackowski J. Caries and periodontal disease in patients with Parkinson's disease. Spec Care Dentist 2011;Sep-Oct;31(5): 178-81.

\section{CORRESPONDENCIA}

Jonathan Harris Ricardo.

Correo electrónico: j.harris.r@hotmail.com 